\title{
A case study of random field models applied to thin-walled composite cylinders in finite element analysis
}

\author{
V. De Groof \& M. Oberguggenberger \\ Institut für Grundlagen der Technischen Wissenschaften \\ Universität Innsbruck, Innsbruck, Austria \\ H. Haller \\ INTALES GmbH \\ Natters, Austria
}

R. Degenhardt \& A. Kling

Institut für Faserverbundleichtbau und Adaptronik

DLR, Braunschweig, Germany

\begin{abstract}
The buckling load of thin-walled structures is known to be greatly affected by the presence of imperfections. These imperfections can be based on e.g. geometry, material properties or loading. It has been shown in the past that including these imperfections by the use of random fields can improve the robustness of finite element models, especially of thin-walled structures. The current random field models are mainly based on the assumption of an exponential correlation structure. Current evaluations of finite element models including random fields are usually done by comparison with the experimental buckling loads. Since it is impossible to include all the sources of imperfections in a finite element model, it is also impossible to single out the performance of the random field model to represent one particular imperfection field. In contrast to current practice, we propose to make a fair assessment of the quality of a random field model by comparing its predictions with the failure loads of the finite element model instead of comparing them directly to the experimental buckling loads. This method will be used to assess the quality of correlation functions and its sensitivities w.r.t. e.g. the number of eigenvectors included. Additionally, a method based on principal component analysis is presented and evaluated using the proposed methodology. Analysis of the limit load resulting from a finite element Monte Carlo simulation using different correlation models showed that the standard correlation models can underestimate the buckling load reduction when not enough eigenvectors are taken into account. When the statistical properties of the random fields have converged, the failure load might still be affected by including additional eigenvectors. This makes it hard to predict the behavior of random fields based on covariance functions when reducing the computational effort by reducing the number of eigenvectors. The random fields based on principal component analysis show better performance at a lower computational cost.
\end{abstract}

\section{INTRODUCTION}

Thin-walled structures are widely spread in industry due to their efficient load-bearing capabilities. Unfortunately, the buckling load of these structures, a failure mode that is often catastrophic, is highly sensitive to structural imperfections from e.g. geometry, material properties, boundary conditions, etc. It is nowadays accepted that the absence of these imperfections in the numerical models share the responsibility for the discrepancies between experiments and numerical predictions (Imbert 1971, Arbocz 2000). To include the effects of imperfections in current deterministic models, knock-down factors are used. Knock-down factors are based on experiments, sometimes executed as early as during the 1960's, and tend to include the effects of all present imperfections in a lumped way (Arbocz \& Starnes Jr 2002, Nemeth \& Starnes Jr 1998). This renders the reduction of the knock-down factor due to minimization of uncertainties of certain variables impossible. It is known that this leads to overly conservative designs and that these knockdown factors do not take the deviant behavior of composite shells into account. In this day and age, finite element software is a widely spread and accepted tool 
in structural analysis. To improve our understanding of the buckling behavior of the structure under investigation, detailed information of the imperfections is required (Schenk \& Schuëller 2005). Based on this information, a probabilistic framework can be created to represent imperfections based on random fields (Elishakoff \& Ren 1999, Stefanou 2009). Random fields have been used in the past to model imperfect geometries, material properties or loading (Bayer \& Roos 2008, Broggi, Calvi, \& Schuëller 2009). Research has been primarily based on exponential covariance functions that were fitted to measurement data in a limited number of cases.

It is our opinion that a fair assessment of these models is lacking as they are usually compared to experimental data that tend to be different from the numerical models due to numerical errors and the exclusions of certain imperfections. In contrast to this approach, the evaluations of the models in this contribution will be assessed with respect to the limit loads obtained from the measured imperfections applied to the finite element model. The differences in failure loads are thus isolated from other uncertainties, numerical errors or imperfections and originate solely from the modeling of the random fields.

It is the goal to report on the results of this assessment with real life data and propose some improvements to keep the computational effort within boundaries. Section 2 will present the theory used to create the stochastic framework. In section 3 the numerical model and the measurement data will be presented. The results of the investigations will be presented in section 4 , and section 5 will conclude on the findings.

\section{THEORY}

Based on experimental data the covariance functions are derived and used to assemble a covariance matrix. Using the Karhunen-Loève expansion, random fields are generated and analyzed with a direct Monte Carlo approach. In a similar way, principal component analysis is used.

\subsection{Covariance functions}

The covariance function contains the spatial variability information of a random field and is used to assemble a covariance matrix that can later be expanded into orthogonal functions (Ghanem \& Spanos 1991). In the past, a variety of covariance models have been applied. Unfortunately, the verification of these functions is often lacking, possibly due to lacking experimental data.

In theory, every function that is symmetric and positive definite can be used to assemble a covariance matrix. Up until now, the exponential covariance function has been the favorite as it is believed to give a good representation of reality. Because of the nondifferentiability of the exponential kernel at zero, improvements have been proposed that alleviate this problem (Spanos, Beer, \& Red-Horse 2007). The standardized exponential and Gaussian covariance functions are given by resp. eq. 1 and 2:

$$
\begin{aligned}
& C(h)=\sigma^{2} \cdot \exp \left(-\frac{|h|}{L_{c}}\right) \\
& C(h)=\sigma^{2} \cdot \exp \left(-\frac{|h|^{2}}{L_{c}^{2}}\right)
\end{aligned}
$$

Here, $L_{c}$ represents the correlation length and $h$ is the lag between two points. Since it is often desired to represent orthotropic random fields, with different correlation lengths in different directions, a product form of the correlations in the different directions is often used:

$C(r, \theta)=\sigma^{2} \cdot c_{r}(r) \cdot c_{\theta}(\theta)$

An advantage of choosing a covariance function is that one does not need specific data to the current geometry to assemble a covariance matrix and generate the random fields.

\subsection{The Karhunen-Loève expansion}

The Karhunen-Loève expansion has been widely used to generate random fields (Bucher 2009), next to alternatives such as e.g. the turning band method (Fenton 1990). Applying the Karhunen-Loève expansion on a stochastic process $U$ decomposes this process into a set of orthonormal functions:

$$
U(x, \omega)=g(x)+\sum_{n=0}^{\infty} \sqrt{\lambda_{n}} \xi_{n}(\omega) \phi_{n}(x)
$$

where $\xi_{n}(\omega)$ is a sequence of orthogonal random variables, $g(x)$ represents a mean function and $\lambda_{n}$ and $\phi_{n}(x)$ represent the eigenvalues and eigenvectors respectively of the following integral equation:

$\int_{W} C\left(x_{1}, x_{2}\right) \phi_{n}\left(x_{2}\right) d x_{2}=\lambda_{n} \phi_{n}\left(x_{1}\right)$

In equation (5), $C\left(x_{1}, x_{2}\right)$ represents the covariance kernel and $\mathrm{W}$ is the domain of the random field. Because the covariance matrix is positive definite and symmetric, the eigenfunctions are orthogonal and its eigenvalues will be real. After discretization, using a Galerkin procedure, equation (5) can be reduced to a generalized eigenvalue problem that can be solved numerically. A useful property of the Karhunen-Loève expansion is that it converges in the mean square sense. Truncation after $M$ terms can considerably reduce the computational effort and will give the smallest mean square error. During the following investigations, it will be assumed that the process $U$ can be approximated by a Gaussian process. 


\subsection{Principal component analysis}

Principal component analysis is used as a statistical technique to decompose a set of observations into its most important modes that are responsible for the main part of its variance. Similarly to the KarhunenLoève expansion, these modes can be truncated and used to simulate a random field with identical statistical distribution as the observed imperfections. The empirical covariance matrix can be calculated by:

$C\left(x_{i}, x_{j}\right)=E\left[U\left(x_{i}\right), U\left(x_{j}\right)\right]-\mu_{i} \cdot \mu_{j}$

Here, $\mu_{i}$ represents the mean value over the set of measurements at a point $x_{i}$.

Principal component analysis is widely used in computational statistics such as data mining and image compression. The main disadvantage of this method over the use of a covariance function is that one needs measurement data to perform the principal component analysis, whereas the Karhunen-Loève expansion as presented previously offers more flexibility.

\subsection{Monte Carlo simulation}

A sample of $N$ random fields was created using a direct Monte Carlo approach. The direct Monte Carlo approach was chosen over other approaches such as Latin Hypercube Sampling because it allowed for easy implementation with the generation of the random fields and can be readily integrated with available, deterministic, finite element packages such as Abaqus or Marc that are able to perform non-linear analyses (Sachdeva, Nair, \& Keane 2007). The mean error resulting from a Monte Carlo simulation can be estimated by:

$e_{M C} \sim \sqrt{\frac{1}{N}}$

This shows that the error decreases independently of the number of variables that are included. This is a highly beneficial property of the Monte Carlo simulation when dealing with big models and a high number of variables to be investigated.

\section{NUMERICAL ANALYSIS}

To evaluate the effects of the random fields a nonlinear finite element buckling analysis was executed using the commercial finite element package Abaqus. This section will go deeper into the properties of the finite element model and the imperfection data.

\subsection{The finite element model}

The cylinders that were measured and tested have the following dimensions: a height of $500 \mathrm{~mm}$ and a radius of $250 \mathrm{~mm}$ (Tab. 1). To the average radius, the
Table 1: Design geometrical and lay-up properties of the cylinders.

\begin{tabular}{lc} 
ders. & \\
\hline Property & Value \\
\hline Radius & $250 \mathrm{~mm}$ \\
Height & $500 \mathrm{~mm}$ \\
Thickness & $0.5 \mathrm{~mm}$ \\
Layers & 4 \\
Layup & $24^{\circ} /-24^{\circ} / 41^{\circ} /-41^{\circ}$ \\
\hline
\end{tabular}

Table 2: Average local radius and standard deviation over the samples within each set.

\begin{tabular}{llll}
\hline Set & Sample Size & Mean $[\mathrm{mm}]$ & Standard Deviation $[\mathrm{mm}]$ \\
\hline Set 1 & 3 & 250.78 & 0.321 \\
Set 2 & 6 & 250.73 & 0.068 \\
\hline
\end{tabular}

imperfections were added. The laminate thickness of only $0.5 \mathrm{~mm}$ causes the cylinders to be very imperfection sensitive. Boundary conditions are applied at both ends of the model, allowing the rotational degrees of freedom at both ends of the cylinder to move. One translational degree of freedom at one end of the cylinder is left free to apply the compressive load.

Since the evaluation of the probabilistic model is done with reference to the measured imperfections applied to the same structural model and the main goal of the study is to evaluate the quality of random field models, boundary conditions could be simplified. A mesh convergence analysis was executed to evaluate the mesh size and the element to be used. It was concluded that a conventional linear, largestrain element with four nodes and reduced integration scheme from the Abaqus element library and a mesh with around 11.000 elements provided a good balance between computational effort and accuracy.

A nonlinear finite element analysis was performed to analyze the stability behavior of the cylinders under investigation. Due to the high number of model evaluations necessary to perform the Monte Carlo analysis, it is necessary to reduce the user input during the analysis by automating the process as much as possible. Therefore, a load-driven arc-length procedure was used to evaluate the load-bearing capacity of the structure. Failure of the structure was defined by a load drop of $20 \%$ compared to the highest load reached during the previous increments. When this load level was reached, the maximum load reached during the complete analysis was defined as the limit load of the structure. As no post-buckling analysis was performed, the strains remain small and in the elastic regime.

\subsection{Analysis of the measured imperfections}

The geometry of a set of 10 cylinders made of composite material were measured and made available by DLR (Degenhardt, Kling, Bethge, Orf, Kärger, Zimmermann, Rohwer, \& Calvi 2010). Analysis of the measurements allowed to evaluate the local geometrical imperfection of the radius at around 200.000 points per cylinder. Due to the fine mesh, it was necessary to decrease the mesh size for the finite ele- 


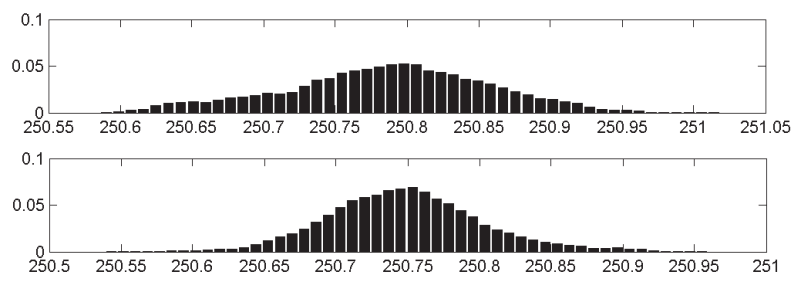

Figure 1: Two histograms representative for the whole sample.

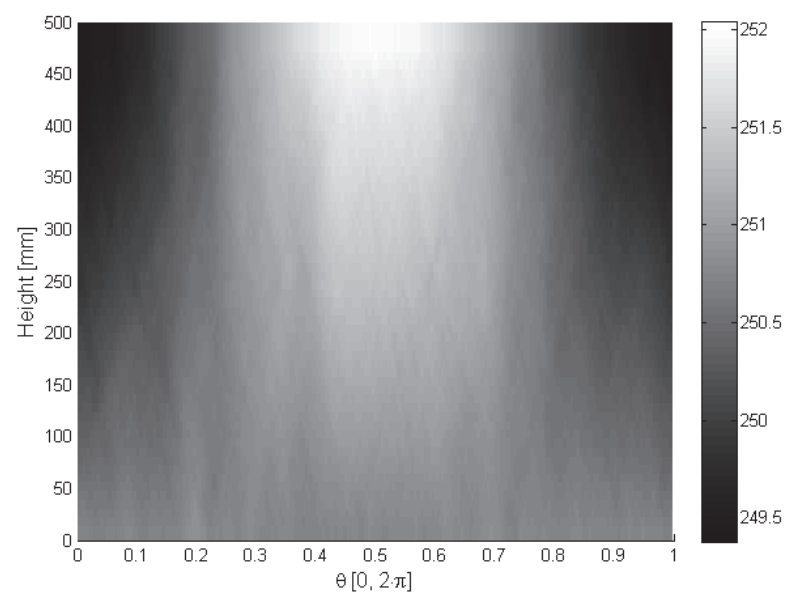

Figure 2: Cylinder which was identified as an outlier and was removed from the set (IGS_Z21). The main deformation mode corresponds with a tilted cylinder.

ment analysis to around 11.000 points. To use these measurements as the basis for a random field analysis, their statistical properties had to be analyzed first. Evaluation of the probability distribution (Fig. 1) shows that the assumption of a Gaussian distribution, although not perfect, is acceptable. Further investigation of the standard deviation and mean showed that 2 sets with distinct properties could be identified (Tab. $2)$. In addition, by visual inspection an outlier was identified which was removed from the set (Fig. 2).
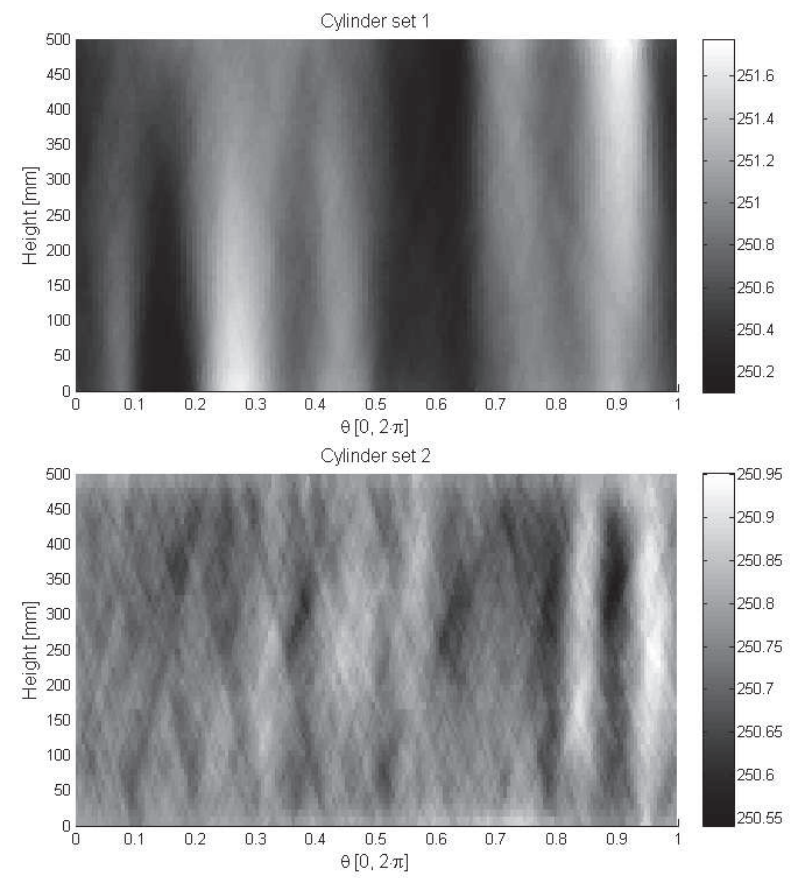

Figure 3: Geometry of 2 example cylinders from the 2 sets. Note the different correlation structures, where the axial correlation of set 1 (IGS_Z17) is higher than the one of set 2 (IGS_Z26).

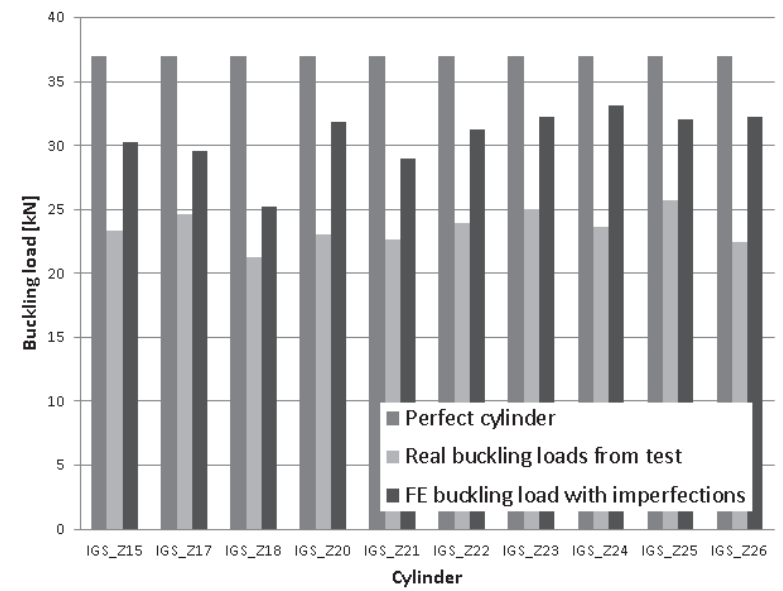

Figure 4: Comparison of the limit load of the ten cylinders. The FE buckling load with imperfections is used in this study as the reference limit load.

Table 3: Overview of the weight and number of eigenvectors at different points.

\begin{tabular}{lc}
\hline Set 1 & \\
Number of eigenvectors & Total weight of eigenvalues \\
\hline 103 & $92 \%$ \\
298 & $98 \%$ \\
500 & $99.12 \%$ \\
1000 & $99.62 \%$ \\
1500 & $99.78 \%$ \\
\hline Set 2 & \\
Number of eigenvectors & Total weight of eigenvalues \\
\hline 153 & $86 \%$ \\
500 & $95.18 \%$ \\
1000 & $97.81 \%$ \\
1500 & $98.74 \%$ \\
\hline
\end{tabular}

The identification of the sets also allowed to separate the correlation structures of both sets as they also revealed to be different, which can be seen in figure 3 . The first, smaller set shows a considerably larger standard deviation of the imperfections as well as a stronger correlation in the axial direction than the second set. Unfortunately, due to limited knowledge of the production process we were unable to identify the reason behind the existence of these 2 sets. As is often the case, the correlation in axial direction is higher as the correlation in radial direction. Earlier investigations of a variety of different correlation functions showed that the effects of different covariance functions are limited (De Groof, Oberguggenberger, Haller, Degenhardt, \& Kling 2012), so it was decided to only use an exponential kernel during this analysis.

\section{RESULTS}

The methodologies described earlier are evaluated and compared to the results obtained from the real measurements. Figure 4 shows the different reference values; the finite element solution without imperfections, the finite element solution with the measured imperfections and the experimental buckling load. To remove the discrepancies inherent to modeling, the following comparisons are made to the finite element solution with the measured imperfections. 
(a)

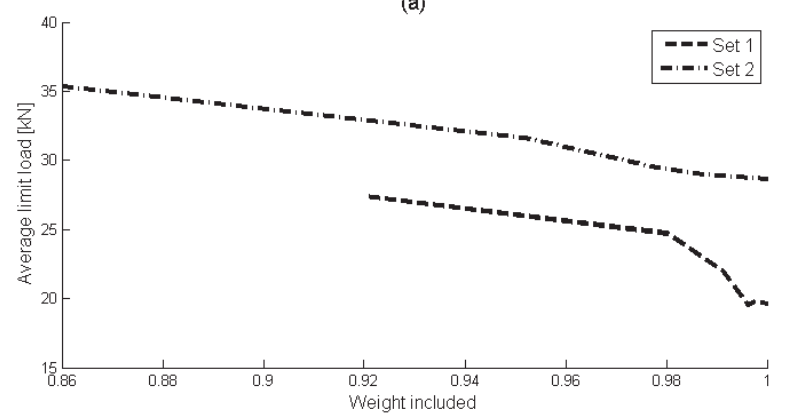

(b)

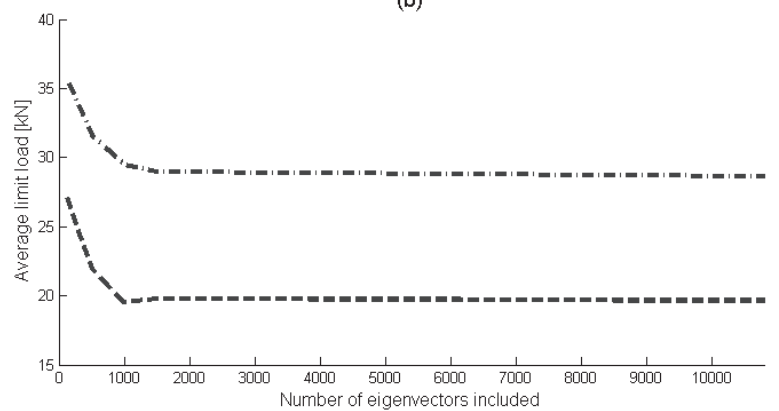

Figure 5: Figure showing the dependence of the limit load on the small eigenvectors. (a) uses the summed weight of the included eigenvalues as a measure, (b) only uses the number of eigenvectors included in the truncation.

\subsection{Karhunen-Loève Expansion}

To make the Karhunen-Loève expansion, the covariance matrix is assembled using an orthotropic, exponential covariance kernel. As explained earlier, based on the values of the eigenvalues, it is possible to truncate the expansion and reduce the computational effort involved in solving the eigenvalue problem considerably. However, the question when to truncate the expansion rises. Therefore, a study of the effect of the small eigenvectors was performed. At different truncation values, the average failure load of a sample of size 100 was calculated. For an overview, see table 3.

As is shown in Fig. 5, using the weight of the eigenvalues can be a misleading guideline. A few 10ths of a percent difference can reduce the average buckling load considerably. Especially for a structure with a high correlation, a lot of the weight is concentrated in the largest eigenvalues. These eigenvalues are linked to the low frequency modes in the Karhunen-Loève expansion. The structure under investigation is, due to its material, failure mode and slenderness, highly sensitive to small imperfections. Using only a small number of eigenvectors, which might be sufficient according to weight of the eigenvalues, can omit these important frequencies. Using the number of largest eigenvalues in a convergence analysis provides a more predictable convergence behavior as using the weight of the largest eigenvalues.

\subsection{Principal Component Analysis}

Another approach is to apply a principal component analysis on the measurement data. The principal component analysis connects the measurement data di-
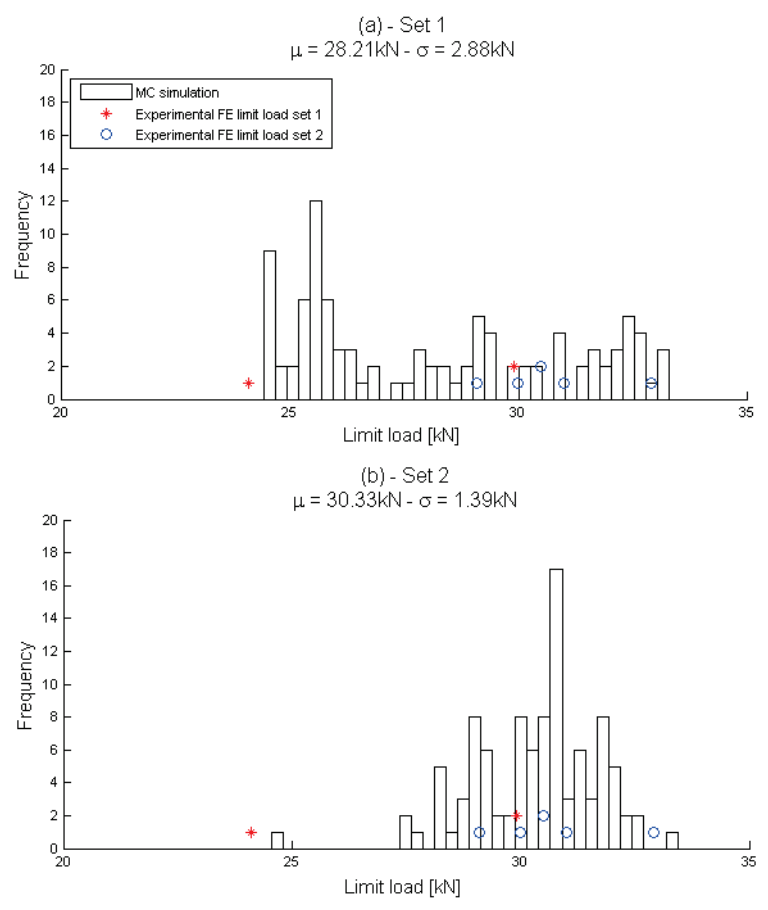

Figure 6: Results of the Monte Carlo simulation using the principal components of the two sets to assemble the random fields. (a) uses the components to simulate set 1 , (b) uses the components of set 2 .

rectly to the covariance matrix. Hence, assuming that the measurements are a valid and complete representation of the main characteristics of the imperfections is paramount. The results of the Monte Carlo simulation (sample size 100) are shown in figure 6.

From the figure, it becomes clear that the principal component analysis can give a good representation, under the condition that the sample size of the measurements is large enough. Knowing more of the production process will increase the understanding of the origin of the principal components. One could e.g. investigate the correlation between the failure load and the weight of the component in the random field. This was done in the current study, but no correlations could be found between a specific component and the failure load.

\subsection{Artificial local imperfections}

As was shown earlier in this contribution, there exists a high correlation between increasing the number of eigenvectors included in the Karhunen-Loève expansion and the limit load. Solving the eigenvalue problem is computationally expensive, especially if it needs to be solved for a high number of eigenvectors including small eigenvalues. Since these small eigenvalues can be linked to the small imperfections, the question was raised if these small imperfections can be added manually without the need of solving an eigenvalue problem.

The approach evaluated here consists of solving the eigenvalue problem for a limited number of eigenvectors - so the truncated Karhunen-Loève expansion simulates the smooth imperfections - and to add the local imperfections manually. These local imperfec- 


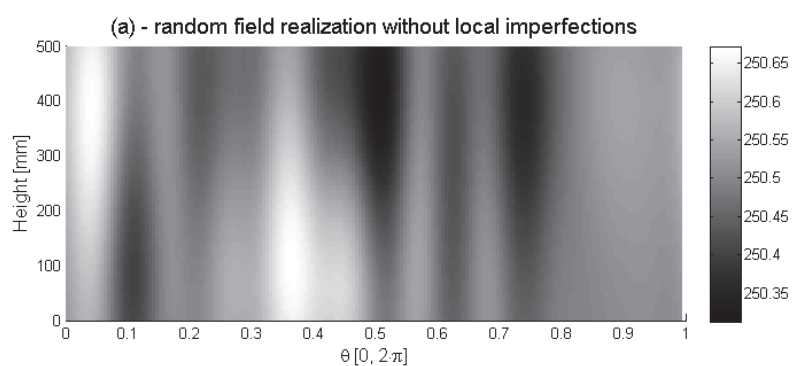

(b) - random field realization with local imperfections

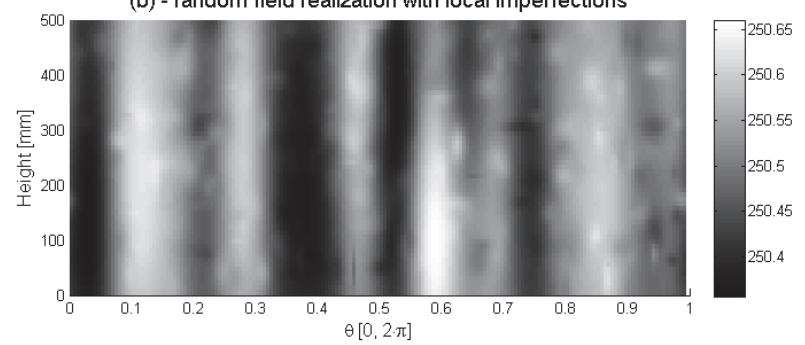

Figure 7: Figure comparing the effect of adding smooth localized imperfections. (a) shows a random field with 30 eigenvectors. (b) uses an identical number of eigenvectors with localized imperfections added.

tions are simulated by small two-dimensional Gaussian shape functions. The size, location and amplitude of the imperfections are randomized and repeatedly added to the smooth random field created with the Karhunen-Loève expansion. The amplitude of the local imperfections also takes the amplitude of the random field into account, so excessive imperfections are not present. Figure 7 shows a random field with and without the localized imperfections.

Generating these localized imperfections evidently requires some knowledge of the process to be modeled to determine limits for the size and amplitudes of the small localized imperfections. This approach is motivated by the observation that the buckling is initiated locally. It combines a realistic geometrical im-
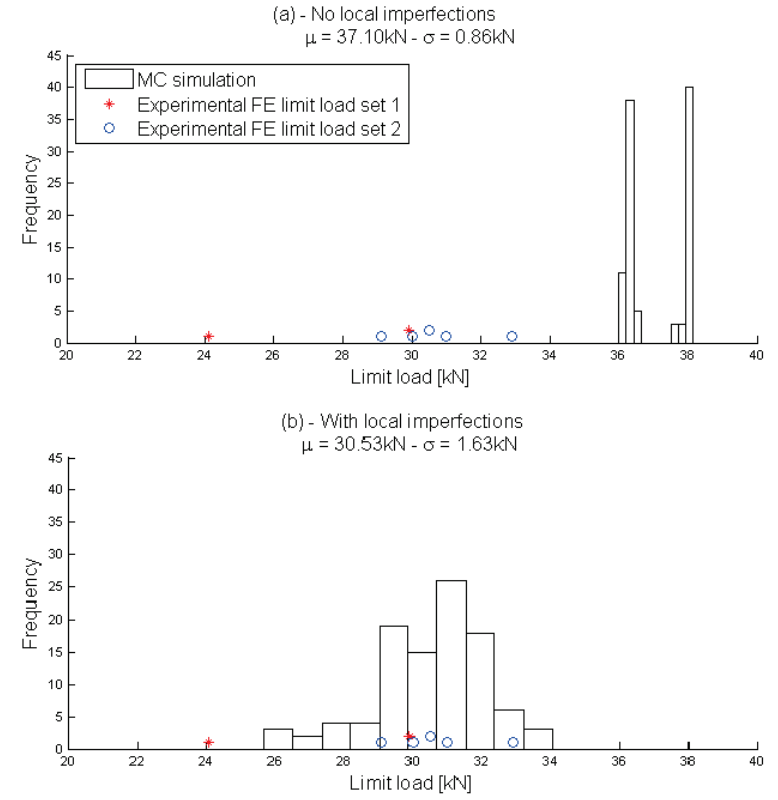

Figure 8: Results of the Monte Carlo simulation using only 30 eigenvectors to simulate the main random field. (b) enriching the smooth random field with small, localized imperfections. perfection with local, artificial initiators (Winterstetter \& Schmidt 2002). As the results show in figure 8, adding smooth localized imperfections can reproduce the buckling behavior correctly. This is noteworthy as the generated imperfections are not larger in size as the original measured imperfections.

\section{CONCLUSIONS}

In this paper it was shown that the use of the Karhunen-Loève expansion to model geometrical imperfections can be misleading. Especially the effect of truncating the expansion can have a large effect on the response. The effect of small changes in the random field model on the structural model might be amplified due to the nonlinearity and can be larger as the statistical indicators show.

An alternative and more effective way is the use of a principal component analysis. This approach works well under the assumption that measurement data is available and its sample size is large enough which might also render its use impractical.

To reduce the computational cost of the eigenvalue problem, an alternative approach combining smooth random fields with artificial localized imperfections is evaluated. The localized imperfections initiate the localized buckling mode that was observed in the nonsmooth random fields and reduce the computational cost considerably. The amplitude was one of the variables that was randomized, but truncated so imperfections could not grow larger as observed in the measured samples. Results show that this approach can reproduce the behavior of the measured samples, but it also requires a higher degree of experience as the user needs to create realistic localized imperfections.

As an important conclusion, we state that the validation of stochastic models is necessary before applying them to engineering problems. Comparing results directly with experimental buckling loads might be misleading due to discrepancies between the model and reality. Especially for shell structures, these discrepancies can result in misleading conclusions. Therefore, it is proposed to evaluate the stochastic model by direct comparison with finite element computations using the measured imperfections.

\section{ACKNOWLEDGEMENT}

This work was supported by the Austrian Ministry of Science BMWF as part of the UniInfrastrukturprogramm of the Research Platform Scientific Computing at the University of Innsbruck.

\section{REFERENCES}

Arbocz, J. (2000). The effect of imperfect boundary conditions on the collapse behavior of anisotropic shells. International Journal of Solids and Structures 37(46-47), 6891-6915. 
Arbocz, J. \& J. Starnes Jr (2002). Future directions and challenges in shell stability analysis. Thin-Walled Structures 40(9), 729-754.

Bayer, V. \& D. Roos (2008). Efficient modelling and simulation of random fields. In 6th International Probabilistic Workshop, Darmstadt.

Broggi, M., A. Calvi, \& G. Schuëller (2009, September). Reliability-based knock-down factor for axially compressed composite cylindrical shells with random imperfections. In European Conference on Spacecraft Structures, Materials and Mechanical Testing. ECSSMMT.

Bucher, C. (2009). Computational analysis of randomness in structural mechanics. CRC Press.

De Groof, V., M. Oberguggenberger, H. Haller, R. Degenhardt, \& A. Kling (2012, September). Quantitative assessment of random field models in finite element buckling analyses of composite cylinders. In CD-ROM Proceedings of the 6th European Congress on Computational Methods in Applied Sciences and Engineering. ECCOMAS: Vienna University of Technology.

Degenhardt, R., A. Kling, A. Bethge, J. Orf, L. Kärger, R. Zimmermann, K. Rohwer, \& A. Calvi (2010). Investigations on imperfection sensitivity and deduction of improved knockdown factors for unstiffened cfrp cylindrical shells. Composite Structures 92(8), 1939-1946.

Elishakoff, I. \& Y. Ren (1999). The bird's eye view on finite element method for structures with large stochastic variations. Computer Methods in Applied Mechanics and Engineering 168(1), 51-61.

Fenton, G. (1990). Simulation and analysis of random fields. Ph. D. thesis, Princeton University, Princeton, NJ.

Ghanem, R. \& P. Spanos (1991). Stochastic finite elements: a spectral approach. Springer Verlag.

Imbert, J. (1971). The effect of imperfections on the buckling of cylindrical shells. Ph. D. thesis, California Institute of Technology, Pasadena, California.

Nemeth, M. \& J. Starnes Jr (1998). The nasa monographs on shell stability design recommendations. Technical report, Langley Research Center, Hampton, Virginia.

Sachdeva, S., P. Nair, \& A. Keane (2007). On using deterministic FEA software to solve problems in stochastic structural mechanics. Computers \& structures 85(5), 277-290.

Schenk, C. \& G. Schuëller (2005). Uncertainty assessment of large finite element systems, Volume 24. Springer Verlag.

Spanos, P., M. Beer, \& J. Red-Horse (2007). Karhunen-loéve expansion of stochastic processes with a modified exponential covariance kernel. Journal of Engineering Mechanics 133, 773.

Stefanou, G. (2009). The stochastic finite element method: past, present and future. Computer Methods in Applied Mechanics and Engineering 198(9-12), 1031-1051.

Winterstetter, T. \& H. Schmidt (2002). Stability of circular cylindrical steel shells under combined loading. Thin-Walled Structures 40(10), 893-910. 